\title{
Variations in air and ground temperature and the POM-SAT model: results from the Northern Hemisphere
}

\author{
R. N. Harris \\ College of Oceanic and Atmospheric Sciences, Oregon State University, USA \\ Received: 4 December 2006 - Published in Clim. Past Discuss.: 6 February 2007 \\ Revised: 28 August 2007 - Accepted: 26 September 2007 - Published: 9 October 2007
}

\begin{abstract}
The POM-SAT model for comparing air and ground temperatures is based on the supposition that surface air temperature (SAT) records provide a good prediction of thermal transients in the shallow subsurface of the Earth. This model consists of two components, the forcing function and an initial condition, termed the pre-observational mean $(P O M)$. I explore the sensitivity of this model as a function of forcing periods at time scales appropriate for climate reconstructions. Synthetic models are designed to replicate comparisons between borehole temperatures contained in the global database of temperature profiles for climate reconstructions and gridded SAT data. I find that the root mean square $(R M S)$ misfit between forcing functions and transient temperature profiles in the subsurface are sensitive to periods longer than about 50 years, are a maximum when the period and the 150-year time series are equal and then decreases for longer periods. The magnitude of the $P O M$ is a robust parameter for periods equal to or shorter than the length of this time series. At longer periods there is a tradeoff between the amplitude of the forcing function and the $P O M$. These tests provide guidelines for assessing comparisons between air and ground temperatures at periods appropriate for climate reconstructions. The sensitivity of comparisons between the average Northern Hemisphere gridded SAT record and subsurface temperature-depth profile as a function of forcing period is assessed. This analysis indicates that the Northern Hemisphere extratropical average SAT and reduced temperature-depth profile are in good agreement. By adding modest heat to the subsurface at intermediate periods some improvement in misfit can be made, but this extra heat has negligible influence on the POM. The joint analysis of borehole temperatures and SAT records indicate warming of about $1.1^{\circ} \mathrm{C}$ over the last 500 years, consistent with previous studies.
\end{abstract}

Correspondence to: R. N. Harris

(rharris@ coas.oregonstate.edu)

\section{Introduction}

Analysis of present-day borehole temperature-depth profiles for ground surface temperature (GST) histories is an important source of climate change information (e.g., Pollack and Huang, 2000). Conductive temperature-depth profiles reflect the changing temperature at the Earth's surface superimposed on the upward diffusion of heat from the Earth's deep interior. On the time scale of contemporary climate change the upward diffusion of heat can be considered constant and forms the background thermal regime against which temperature anomalies can be referenced. Changing surface temperature with time gives rise to curvature as a function of depth in the shallow subsurface. The coefficient of thermal diffusivity links depth and time so that temperature anomalies as a function of depth can be transformed to surface temperature variations as a function of time. In this way observations of temperature with depth are transformed to interpretations of GST histories.

Theoretically GST histories can be reconstructed anywhere subsurface heat transfer is conductive and constitute an important dataset in areas where other sources of paleoclimatic information is limited (e.g., Lachenbruch and Marshall, 1986; Taylor et al., 2006). Climate analysis based on borehole temperatures is powerful because it is rooted in the conservation of energy and the physics of heat diffusion. As a result this technique does not suffer from ambiguities due to an empirical calibration between a proxy measurement and temperature. However, a number of non-climatic processes can distort temperature-depth profiles, and perturbations resulting from these processes should be either ruled-out or corrected (see Appendix of Chisholm and Chapman, 1992).

An important question regarding the use of borehole temperature profiles to reconstruct GST histories is resolution. Chisholm and Chapman (1992) considered the resolution of both step functions and linear ramps for the general case where there are two free parameters, the magnitude of the

Published by Copernicus Publications on behalf of the European Geosciences Union. 

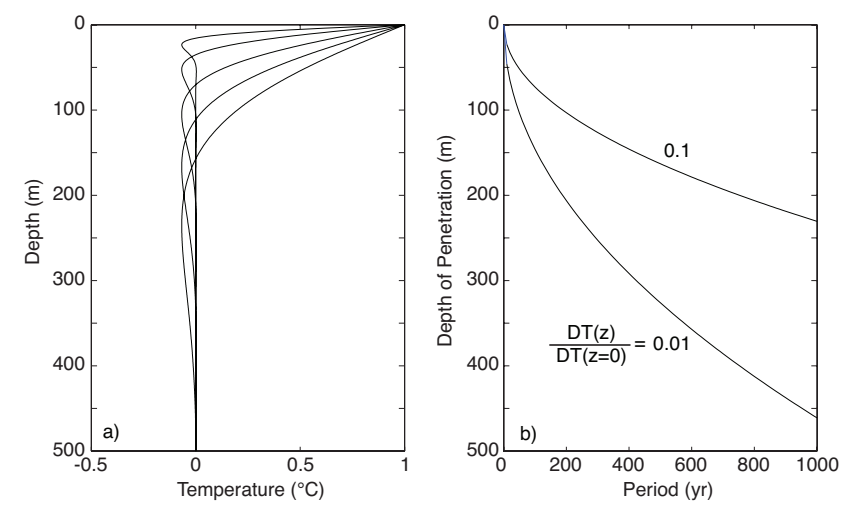

Fig. 1. The frequency dependence of diffusion. (a) Transient temperature profiles constructed from forcing functions with periods of $5,10,50,100,200$, and 500 years. In each case the forcing function has an amplitude of $1^{\circ} \mathrm{C}$ and is phase shifted to reflect recent warming. (b) Depth attenuation as a function of period. The lines show the depth at which the ratio of the subsurface temperature perturbation to the surface amplitude are lost in the background.

change and the onset time of that change. They found that the magnitude of change is much better resolved than the timing of change, consistent with the physics of diffusion. Other resolution studies have come to similar conclusions (Clow, 1992; Beltrami and Mareschal, 1995; Harris and Chapman, 1998a).

The loss of temporal resolution with time in the past can be illustrated through the subsurface diffusion of temperature caused by a periodic surface temperature condition. This process can be described by (e.g., Carslaw and Jaegar, 1959),

$T(z, t)=T_{o}+\Delta T \exp \left(-z \sqrt{\frac{f \pi}{\alpha}}\right) \cos \left(2 \pi f t-z \sqrt{\frac{f \pi}{\alpha}}\right)$

where $T$ is temperature, $T_{o}$ is the mean surface temperature, $\Delta T$ is the amplitude of the surface variation, $f$ is the frequency of the surface wave and $\alpha$ is thermal diffusivity. The exponential term describes the attenuation of a thermal perturbation with depth and shows that the attenuation is proportional to the square root of frequency. High frequency climatic information is lost at relatively shallow depths, while low frequency climatic information penetrates to greater depth (Fig. 1). For example, if the fractional change in surface temperature that can be resolved is 0.01 , a periodic surface temperature variation with a 10 -year period is limited to depths less than $50 \mathrm{~m}$, while a 50 -year period oscillation is limited to depths less than $100 \mathrm{~m}$. Equation (1) shows how the loss of frequency content with depth translates to a loss of temporal resolution with time in the past through the attenuation of high frequency information. Analysis of temperature-depth profiles for climatic change is therefore ideally suited for determining long-term (decadal timescale and longer) trends of surface temperature change.
A second consideration is the resolving power of the depth distribution of temperature measurements to events in the past. Resolution of climatic events decreases with time in the past and is proportional to the maximum depth of the temperature-profile (Clow, 1992). For example, based on the depths of temperature-depth profiles comprising the dataset of global borehole temperature-depth profiles for climatic analysis, this dataset is considered to have resolution of about 500 years in the past (Huang et al., 2000). However, large events occurring before this time have the potential of biasing the results. Finally, it is also important to note that borehole temperature GST reconstructions are not unique.

Most temperature-depth profiles are located in areas where surface air temperature (SAT) records exist and the combination of these datasets offer additional information about our climate system. Comparisons of these datasets, either qualitatively (Huang et al., 2000) or quantitatively (Harris and Chapman, 2001) increases the confidence in GST reconstructions by providing independent evidence of temperature change for the period of overlap, and by helping to place SAT records in a longer context. Long-term regional comparisons at the 100-year time scale generally show good agreement between air and ground temperatures (Huang et al., 2000; Harris and Chapman, 2001; Beltrami, 2002; Pollack and Smerdon, 2004). Modeling studies using General Circulation Models allow comparisons between air and ground temperatures at longer time scales and also suggest good agreement between changes in air and ground temperature (Gonzáles-Rouco et al., 2003, 2006). However, questions regarding changes in the relationship between air and ground temperatures have prompted detailed investigations often in combination with other meteorological parameters (e.g. Baker and Ruschy, 1993; Putnam and Chapman, 1996; Smerdon et al., 2003, 2004, 2006; Bartlett et al., 2006; Chudinova et al., 2006; Stieglitz and Smerdon, 2007). These studies have found that variations in air and ground temperatures generally track each other over the time period of study. More importantly however, these studies illuminate processes that may adversely influence the relationship between air and ground temperatures. Candidate processes include trends associated with cold season snow cover and warm season solar insolation. Other processes such as changing ground cover and soil moisture has also been suggested to adversely affect the relationship between air and ground temperature (Lewis and Wang, 1998; Nitoui and Beltrami, 2005; Pollack et al., 2005; Mottaghy and Rath, 2005). Unfortunately, regional networks documenting these processes are not long enough to unambiguously address this issue at time scales appropriate for borehole climatic studies. Most studies documenting variations between air and ground temperatures are at the annual time scale or are over a few annual cycles, and in the context of GST reconstructions, these high frequencies are attenuated before they can reach depths relevant for centennial scale GST reconstruction histories. Notable exceptions at specific locations where longer time 
series are available include Baker and Baker (2000), Bartlett et al. (2006) and García-Suárez and Butler (2006).

One way to compare air and ground temperatures at long time scales is to diffuse air temperatures into the ground and compare them with measured ground temperatures. This is the essence of the POM-SAT model that is described in the next section. This study explores the sensitivity of the POMSAT model to various forcing frequencies with particular attention paid to the sensitivity of the misfit and the initial condition, the pre-observational mean temperature (POM). Because of the frequency-depth dependence of attenuation and because each potential process affecting the coupling between air and ground temperatures likely has a characteristic frequency it is important to investigate this model as a function of frequency. These issues are explored with a series of numerical tests and then with data from the Northern Hemisphere. In the synthetic tests, a time series representing an SAT record with known frequencies is constructed. Individual frequencies are attenuated and the distorted time series is diffused into the subsurface where it is compared to the true synthetic forcing function. This construction mimics processes that may decouple air and ground temperatures.

\section{The POM-SAT model}

Conceptually, the approach advocated by the POM-SAT model is that we have two independent data sets of surface temperature change, borehole temperature-depth profiles and SAT data, and that we can glean information about our climate system through the judicious comparison of these data sets. It seems reasonable to start with the supposition that these datasets are related, and that SAT data provide a good starting point for unraveling GST histories from temperature profiles.

In a series of papers Harris and Chapman (1998b, 2001, 2005) argued that a good way to quantitatively compare air and ground temperatures at long time scales is to compute a transient temperature profile using the SAT record as a forcing function. In this model, the SAT record is parameterized as a series of annual mean temperatures, $T_{i}$, corresponding to time before the temperature-depth measurements were made, $\tau_{i}$. The transient temperature profile, $\Delta \mathrm{T}_{t}(\mathrm{z})$ can be expressed as (Carslaw and Jaegar, 1959),

$$
\begin{aligned}
& \Delta T_{t}(z)=\left(T_{1}-P O M\right) \operatorname{erfc}\left(\frac{z}{\sqrt{4 \alpha \tau_{1}}}\right) \\
& +\sum_{i=2}^{n} \Delta T_{i} \operatorname{erfc}\left(\frac{z}{\sqrt{4 \alpha \tau_{i}}}\right)
\end{aligned}
$$

where $P O M$ is the initial condition termed the preobservational mean temperature, $\alpha$ is the thermal diffusivity, and erfc is the complementary error function. This equation contains two free parameters, the POM and $\alpha$, and $\mathrm{N}$ fixed $T_{i}$ values. In practice the value of $\alpha$ is usually as- sumed a priori based on laboratory measurements that indicate a value of $1 \times 10^{-6} \mathrm{~m}^{2} / \mathrm{s}$ is a reasonable value (Clauser and Huenges, 1995). Expressing the POM-SAT model in this manner (Eq. 2) shows explicitly that the model consists of two components, the $P O M$ and the forcing function. The $P O M$ is the initial temperature that minimizes the misfit between the reduced temperature profile, (i.e., the anomalous temperatures relative to the background thermal regime) and the transient temperature profile in the least squares sense according to the misfit function,

$S(m)^{2}=\sum_{z=0}^{t l_{2}}\left(\Delta T_{r}-\Delta T_{t}\right)^{2}$.

Here $\Delta T_{r}$ is the reduced temperature profile relative to the surface temperature intercept, $\Delta T_{t}$ is the transient profile relative to the $P O M$, and $t l_{2}=2 \sqrt{4 \alpha \tau}$ is twice the thermal length where $\tau$ is the first annual SAT value relative to the present. If $\tau$ is 150 years, $t l_{2}$ corresponds to a depth of $275 \mathrm{~m}$. At this depth a $1^{\circ} \mathrm{C}$ step change in temperature leads to a $5 \mathrm{mK}$ perturbation in subsurface temperature. Limiting the misfit to a depth of two thermal lengths increases the sensitivity of the misfit by restricting the calculation to the shallow subsurface where sensitivity to surface temperature forcing is the greatest. Below this depth the signal generated from the SAT time series is negligible. The POM-SAT model has significance to the extent that air and ground temperatures faithfully track each other.

In addition to representing a temperature history, the reduced temperature profile and SAT records are also a measure of the change in heat content, $\Delta Q$ (e.g., Beltrami et al., 2002),

$\Delta Q=m c \Delta T$

where $m$ is mass and $c$ is specific heat. These changes in heat content are both relative to a reference temperature, the surface temperature intercept for the reduced temperature profile, and the POM temperature for the SAT record. In this framework, the algorithm adjusts the POM, until the two quantities of heat are in agreement to the extent possible with a single parameter, and in the least squares sense. The magnitude of the least squares misfit gives a measure of how well the temperature histories agree. That is, while we may have the same quantity of heat in the atmosphere and ground, if the temperature history is different, then the least squares misfit may be large and the diffusion model may not be valid. This perspective adds insight into how the POM is optimized.

Advantages of the POM-SAT model are multi-fold (Harris and Chapman, 1998b). First, this model allows a quantitative comparison between SAT records and reduced temperature profiles using the same frequency-depth dependence. These quantitative comparisons are important because they act as a check on the internal consistency of the two data sets, and internal consistency can increase our confidence that 
Table 1. Optimum $P O M$ as a function of missing period.

\begin{tabular}{llll}
\hline Missing Period & True $P O M$ & Optimum $P O M$ & $R M S$ misfit \\
\hline Yr & ${ }^{\circ} \mathrm{C}$ & ${ }^{\circ} \mathrm{C}$ & $\mathrm{mK}$ \\
None & 0 & 0.03 & 12 \\
75 & 0 & -0.04 & 39 \\
150 & 0 & -0.06 & 41 \\
500 & 0 & -0.13 & 25 \\
\hline
\end{tabular}

temperature-depth profiles are being correctly interpreted. In contrast, qualitative comparisons between GST inverse solutions and SAT records may be misleading because of the different frequency content of the two records being compared. Additionally because this formulation solves the heat equation in the forward sense it is very stable, and it minimizes the number of free parameters. In this sense it is the simplest model that fits the reduced temperature profile. Finally, this model is based on the physics of heat diffusion and thus does not solely rely on statistical correlations.

Questions that naturally arise in the use of the POM-SAT model include: how robust is the POM, how sensitive is the model fit to forcing functions, and what does the $R M S$ misfit imply about the relationship between variations in air and ground temperatures? In the sensitivity tests that follow I focus on these questions as a function of forcing amplitude and frequency.

\section{Sensitivity of the POM-SAT model to surface temper- ature forcing}

With the increasing importance of borehole temperature profiles in climate reconstructions it is important to investigate the sensitivity of the POM-SAT model to potential discrepancies in the frequency content of the surface forcing function and the reduced temperature profile. Discrepancies in the frequency content of these two signals might arise from some process that distorts the SAT signal as it enters and diffuses through the subsurface (e.g., Bartlett et al., 2006; Stieglitz and Smerdon, 2007). I explore this issue with a number of synthetic tests. Each test starts with the construction of a surface forcing function. This function is then distorted in amplitude or phase or both for a particular period and diffused into the subsurface. The resulting transient temperature profile is then compared with the original, undistorted, forcing function, and the POM that produces the minimum misfit is determined. These comparisons provide an indication of the sensitivity of a particular forcing period and the $P O M$ through the root mean square (RMS) misfit. The RMS misfit is calculated with

$$
R M S=\sqrt{\frac{1}{n} S(m)^{2}}
$$

where $S(m)$ is the least squares misfit function (Eq. 3) and $n$ is the number of data points to a depth of two thermal lengths.

In the first set of synthetic tests, the surface forcing function has a duration of 500 years and consists of 75, 150, and 500 year periods, each with an amplitude of $0.2^{\circ} \mathrm{C}$ (Fig. 2a). All components of the forcing function are phase shifted to produce a maximum warming at present of $0.6^{\circ} \mathrm{C}$ above the $P O M$ of $0^{\circ} \mathrm{C}$. Any period could be investigated, but 500 years corresponds to the temporal resolution of GST reconstructions formed from the temperature profiles in the global data base (Huang et al., 2000), 150 years corresponds to the time span of globally available SAT data relative to 2001, the time for which the reduced temperature profiles have been standardized (Harris and Chapman, 2001), and 75 years is an even fraction of 150 years. The $P O M$ is set to $0^{\circ} \mathrm{C}$ and the true surface forcing is diffused into the ground to produce the true transient (Fig. 2b). The RMS misfit between the true and synthetic transient is plotted in Fig. 2c. I investigate the impact of only using the past 150 years of the forcing function and successively muting the 75,150 , or 500 year period from the forcing function (Fig. 2a). The 150-year forcing function with no missing periods has a $P O M$ of $0.02^{\circ} \mathrm{C}$ and an $R M S$ misfit of $12 \mathrm{mK}$ (Table 1). The $P O M$ has a slight positive bias that balances the missing portion of the forcing function. In contrast, the forcing function with muted periods contain less subsurface heat than the true transient (Fig. 2b) and the POM compensates with a negative bias. The bias in $P O M$ increases the effective heat content of the distorted surface forcing to minimize the misfit with the true surface forcing. This set of simulations demonstrates several features of the POM-SAT model. First, the change in POM from the true value is significantly less than $0.2^{\circ} \mathrm{C}$ supporting the contention that the $P O M$ is a robust parameter (Table 1). Secondly, the POM is a well-determined parameter as indicated by the sharp trough in the RMS misfit function (Fig. 2c). Finally, there is a potential danger is taking a particular model fit as evidence of faithful tracking between air and ground temperatures. For each comparison the minimum $R M S$ appears small even though there is a significant discrepancy between the forcing function and the true synthetic.

In practice several processes may degrade relationships between air and ground temperatures in two ways. First, processes distorting the surface forcing may not entirely mute a specific frequency but only attenuate it, and may also affect the phase. Secondly, reduced temperature profiles rarely start at the surface and because of the frequency dependence of diffusion this decreases the sensitivity of the model to the data. In the following numerical experiments I extend the previous analysis by investigating the robustness of the $P O M$ and $R M S$ misfit under these conditions.

In the second set of tests the true surface forcing contain periods of $25,50,75,150$, and 500 years, each with an amplitude of $0.2^{\circ} \mathrm{C}$ and phase shifted to produce recent warming of $1^{\circ} \mathrm{C}$ (black line, Fig. 3a). As in the previous set of experiments, the true forcing function has a duration of 500 years, 

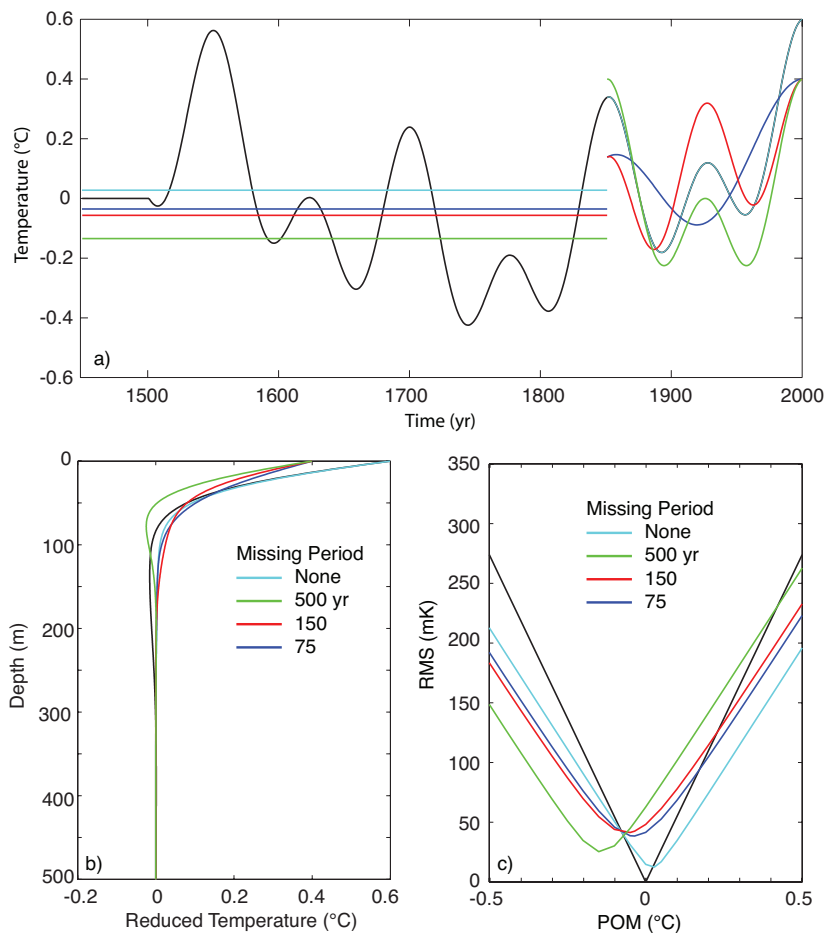

Fig. 2. Sensitivity of the POM-SAT model to a missing period. (a) The true surface forcing function (black line) is composed of a linear combination of surface forcings having periods of 75,150 , and 500 years. Each period has an amplitude of $0.2^{\circ} \mathrm{C}$. The forcings have been phase shifted to show recent warming of $0.6^{\circ} \mathrm{C}$. Horizontal lines shows synthetic forcings with individual periods muted, and dashed lines show their best fitting POM. (b) Transient temperature profiles constructed from surface forcing (black) and best fitting synthetic forcings. (c) $R M S$ misfit as a function of $P O M$ for each comparison.

and the true $P O M$ is $0^{\circ} \mathrm{C}$. The resulting true transient (black line, Fig. 3b) has an amplitude at the surface of $1{ }^{\circ} \mathrm{C}$. Distorted transients are constructed using only the first 150 years before present and by varying the amplitude and phase of the 150-year period and diffusing the surface forcing into the subsurface. For the 150-year period the minimum $R M S$ corresponds to a forcing function amplitude of $0.2^{\circ} \mathrm{C}$ (Fig. 3c), and a $P O M$ of $0^{\circ} \mathrm{C}$ (Fig. 3d), as expected. More generally, Figs. $3 \mathrm{c}$ and $3 \mathrm{~d}$ show how the $R M S$ and $P O M$ misfit vary as a function of errant amplitudes and phases associated with the 150 -year period. For example, the 150 -year period with amplitudes of $1^{\circ}$ and $-1^{\circ} \mathrm{C}$ are shown (Fig. 3a). The synthetic forcings are diffused into the subsurface and the POMs optimizing the misfits are determined. These examples show how the heat content can be made equivalent with the POM temperatures, but that the $R M S$ misfits are increased (Fig. $3 \mathrm{c}$ ). The $P O M$ temperatures shift so that the effective heat content of the distorted transients best matches the true transient. The $R M S$ misfit is generally more sensitive to the amplitude than the phase, although sensitivity decreases with phase and is
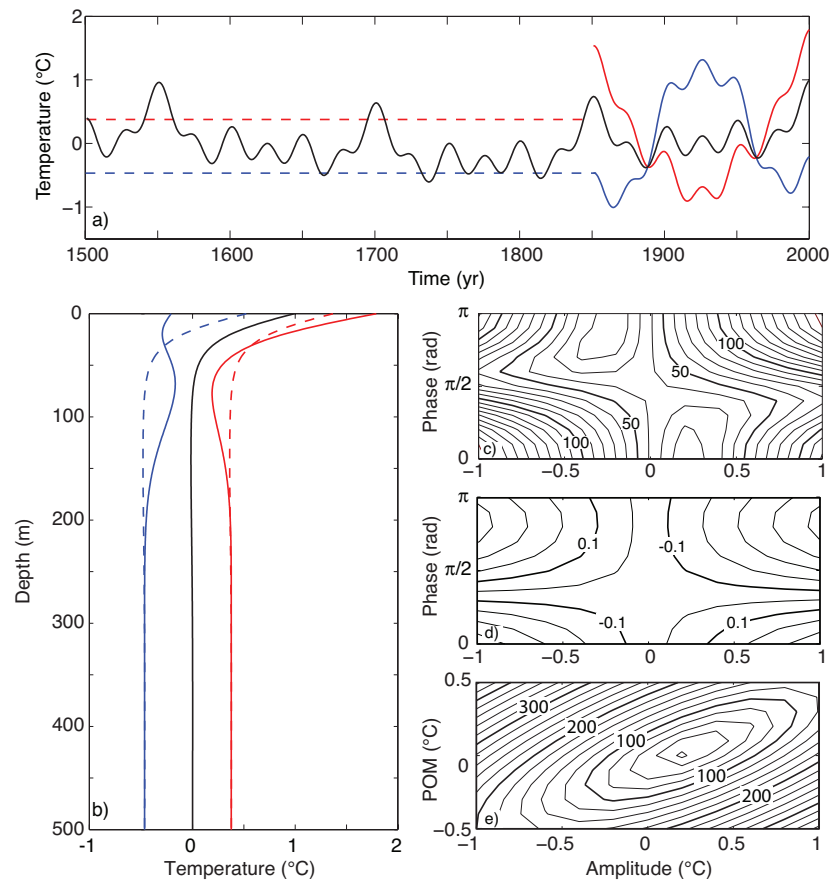

Fig. 3. Sensitivity of the $P O M$ model as a function of forcing function amplitude and phase for the 150 year period. (a) True surface forcing function (black line) composed of a linear combination of sinusoids having periods of $25,50,75,150$, and 500 years, each with an amplitude of $0.2^{\circ} \mathrm{C}$. The forcings have been phase shifted to produce recent warming of $1.0^{\circ} \mathrm{C}$. Red and blue lines show example surface forcing where the amplitude of the 150 year period is $1.0^{\circ}$ and $-1.0^{\circ} \mathrm{C}$, respectively, and the phase is $0 \mathrm{rad}$. Dashed lines show the best fitting POM. (b) Transient temperature profiles constructed from surface forcing (black) and example forcings (red and blue lines). For comparison, both the reduced and transient temperature profiles are offset by their respective POMs. (c) $R M S$ misfit as a function of forcing amplitude and phase of the 150 year period. The contour interval is $10 \mathrm{mK}$. (d) $P O M$ as a function of the forcing amplitude and phase of the 150 year period. The contour interval is $0.1^{\circ} \mathrm{C}$. (e) $R M S$ misfit as a function of forcing amplitude and $P O M$. The contour interval is $20 \mathrm{mK}$.

a minimum when the forcing is approximately $\pi / 2$ out of phase. This loss of resolution occurs because this phase shift puts the misfit deeper in the subsurface and coupled with the effects of attenuation the sensitivity to the misfit function is decreased. If we can neglect the phase, a convenient way to condense this information is to plot the $R M S$ misfit as a function of both the forcing amplitude and POM (Fig. 3e). This panel shows that for the 150 -year period the $R M S$ misfit is sensitive to both the forcing amplitude and $P O M$. As the amplitude of this forcing period increases the POM also increases to offset the extra heat in the ground.

In practice reduced temperature profiles do not start at the surface and contain noise. Both of these attributes influence the sensitivity of the RMS misfit and POM. For comparison with later results, Figs. $4 \mathrm{a}, 4 \mathrm{~b}$, and $4 \mathrm{c}$ repeat the simulations 

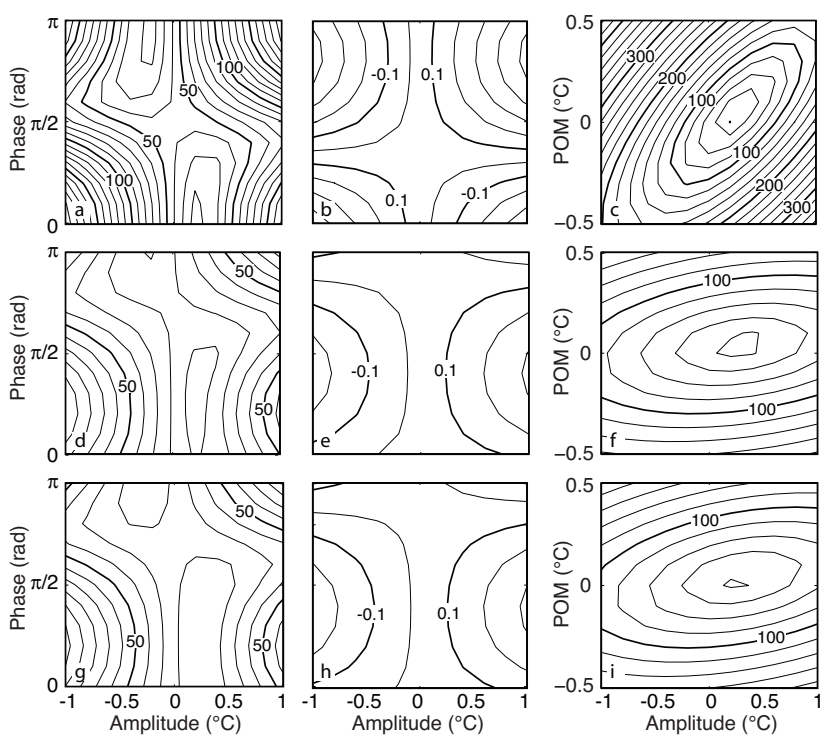

Fig. 4. Left column shows root mean square (RMS) misfit between true and synthetic model as a function of forcing amplitude and phase with a contour interval of $10 \mathrm{mK}$. Middle column shows best fitting $P O M$ as a function of forcing amplitude and phase with a contour interval of $0.1^{\circ} \mathrm{C}$. Left column show $R M S$ misfit between true and synthetic model as a function of forcing amplitude and $P O M$ with a contour interval of $20 \mathrm{mK}$. The forcing function and phase of the 150 year period is varied. The top row shows true reduced temperature profile consists of temperature data from 0 to $500 \mathrm{~m}$ with a spacing of $1 \mathrm{~m}$. This set of experiments are noise free. Middle row shows true reduced temperature profile consists of temperature data from 30 to $500 \mathrm{~m}$ with a spacing of $1 \mathrm{~m}$. This set of experiments are noise free. Bottom row shows true reduced temperature profile consists of temperature data from 30 to $500 \mathrm{~m}$ depth, a measurement spacing of $5 \mathrm{~m}$. $10 \mathrm{mK}$ zero-mean Gaussian noise is added.

shown in Fig. 3. In the second set of simulations the reduced temperature profile starts at a depth of $30 \mathrm{~m}$ (Figs. $4 \mathrm{~d}, 4 \mathrm{e}$ and $4 \mathrm{f}$ ). This decreases the sensitivity to the $R M S$ misfit because of progressive amplitude attenuation with depth and indicates the importance of shallow data for comparisons between subsurface temperature profiles and SAT records. Finally $10 \mathrm{mK}$ zero mean Gaussian noise is added which also decreases the sensitivity of the RMS misfit function (Figs. $4 \mathrm{~g}$, $4 \mathrm{~h}$ and $4 \mathrm{i}$ ). For these tests, the $R M S$ misfit is more sensitive to the missing shallow temperature data than the modest amount of noise added to the subsurface temperatures.

Figure 5 shows the RMS misfit as a function of forcing amplitude and $P O M$ for each of the investigated periods. The reduced temperature profile extends between depths of 30 and $500 \mathrm{~m}$, the measurement spacing is $5 \mathrm{~m}$ to match the characteristics of the reduced temperature profile constructed for the Northern Hemisphere (Harris and Chapman, 2001). Additionally $10 \mathrm{mK}$ of zero mean Gaussian noise is added. The minimum $R M S$ misfit varies between 12 and $14 \mathrm{mK}$. If we assume that a $10 \mathrm{mK}$ change in the misfit function is significant, then these comparisons are relatively insensitive to forcing periods less than about 50 years. This lack of sensitivity is due to the frequency filtering and to a lesser extent on the way the $R M S$ is calculated. For reasonable amplitudes, high frequency perturbations are filtered out before they can have a significant effect on the transient temperature profile and $R M S$ misfit. In contrast these comparisons are modestly sensitive to forcing periods of 75, 150 and 500 years.

For all periods less than or equal to the length of the forcing function of 150 years the POM is a relatively robust parameter independent of the amplitude of the forcing period. For example, an errant amplitude of $1^{\circ} \mathrm{C}$ associated with the 150 -year period leads to an error in the $P O M$ of only $0.1^{\circ} \mathrm{C}$. However, the $P O M$ becomes increasingly sensitive to periods longer than the time series. For a 500-year period an errant amplitude of $1^{\circ} \mathrm{C}$ would produce an error in the $P O M$ temperature of $0.7^{\circ} \mathrm{C}$. This result illustrates that for long periods there is a tradeoff between forcing function amplitude and the magnitude of the POM. These results indicate that very low frequency discrepancies between the forcing function and reduced temperature profile have the potential to bias the POM. However the misfit function provides a tool for assessing goodness of fit at these lower frequencies.

\section{Data}

To investigate the relationship between the average of temperature-depth profiles and SAT records at various frequencies, I focus on the Northern Hemisphere where most of the temperature-depth profiles exist (Fig. 6a). Temperaturedepth profiles come from the global database for climate change studies (Huang and Pollack, 1998). Attributes of temperature profiles included in the database are described in Pollack and Huang (2000). Requirements for inclusion in this data set include having measurements at least as shallow as $100 \mathrm{~m}$ and at least as deep as $200 \mathrm{~m}$. Additionally the temperature data are required to show a smooth variation with depth and show no evidence for advective disturbances or permafrost.

There are now a number of gridded SAT data sets available each with their own characteristics. The Mitchell and Jones (2005) grid has a spatial resolution of $0.5^{\circ}$ but only extends back to 1901. In contrast the Jones and Moberg (2003) grid has a spatial resolution of $5 \times 5^{\circ}$ and extends back to 1851. Because the focus of this study is on temporal comparisons rather than spatial comparisons, I use the $5 \times 5^{\circ}$ gridded data set of Jones and Moberg (2003). Experiments with the Mitchell and Jones (2005) gridded data set yielded very similar results. Gridded SAT data from grid cells that contain temperature profiles are weighted by area and averaged together. The data cover a time period between 1851 and 2001 (Fig. 6b). 

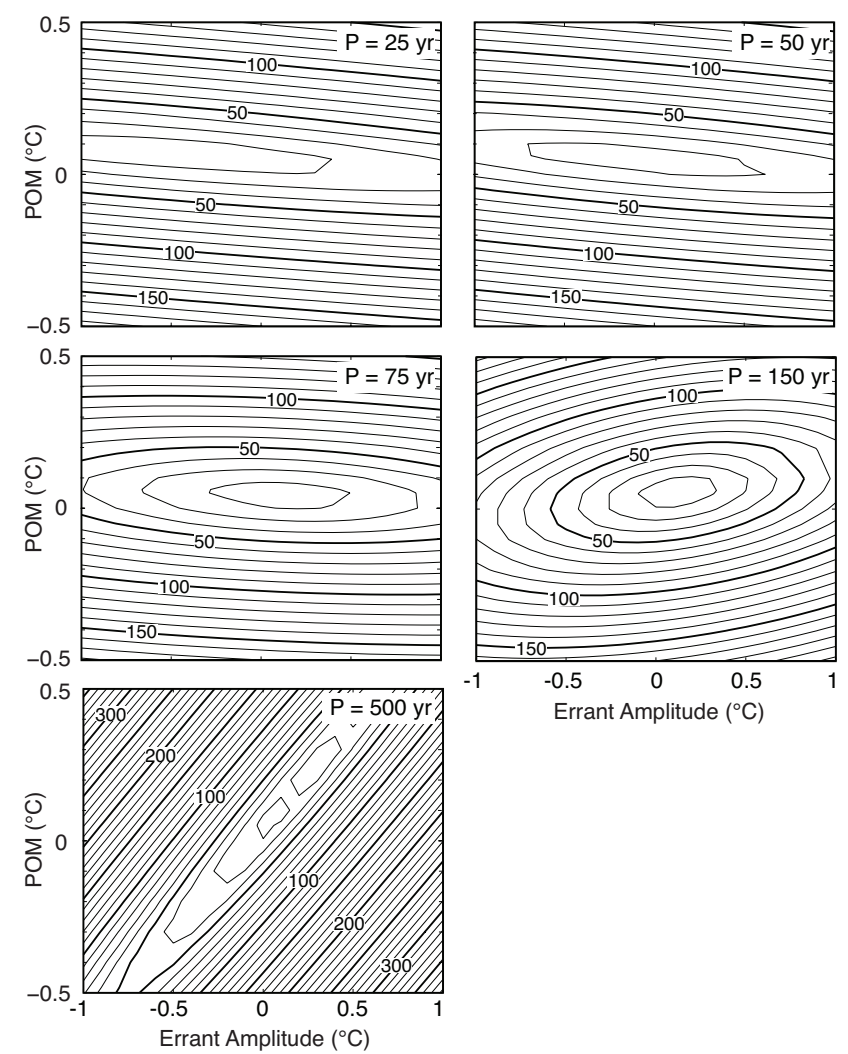

Fig. 5. $R M S$ misfit as a function of forcing amplitude and $P O M$ for different forcing periods. Period, $P$, corresponding to each panel is shown in upper right corner. Contour interval is $10 \mathrm{mK}$.

Temperature-depth profiles are analyzed as described in Harris and Chapman $(2001,2005)$. For each temperature profile, the background thermal field is parameterized in terms of the long-term thermal gradient and mean surface temperature intercept. For consistency these background parameters are estimated for each profile using data below $160 \mathrm{~m}$, a depth dictated in part by the data but also sufficient to minimize perturbations from recent GST variations, while also providing a sufficient depth interval to obtain a robust estimate of these parameters. The background thermal regime is subtracted from each temperature-depth profile to form a reduced temperature profile. This compilation of temperature profiles represents data collected over a 44-year period (1958-2001). These profiles are forward continued in time using a Laplace transform, assuming a constant GST between the year the borehole was logged and 2001 (Harris and Chapman, 2001). This procedure yields conservative and consistent reduced temperature profiles. An average reduced temperature profile is computed by averaging individual reduced temperatures for each $5^{\circ} \times 5^{\circ}$ grid cell containing temperature logs, weighting each grid cell by its area, and then averaging all grid cells together. The mean reduced temperature profile (Fig. $6 \mathrm{~d}$ ) has a magnitude of $0.5^{\circ} \mathrm{C}$ at $30 \mathrm{~m}$ that
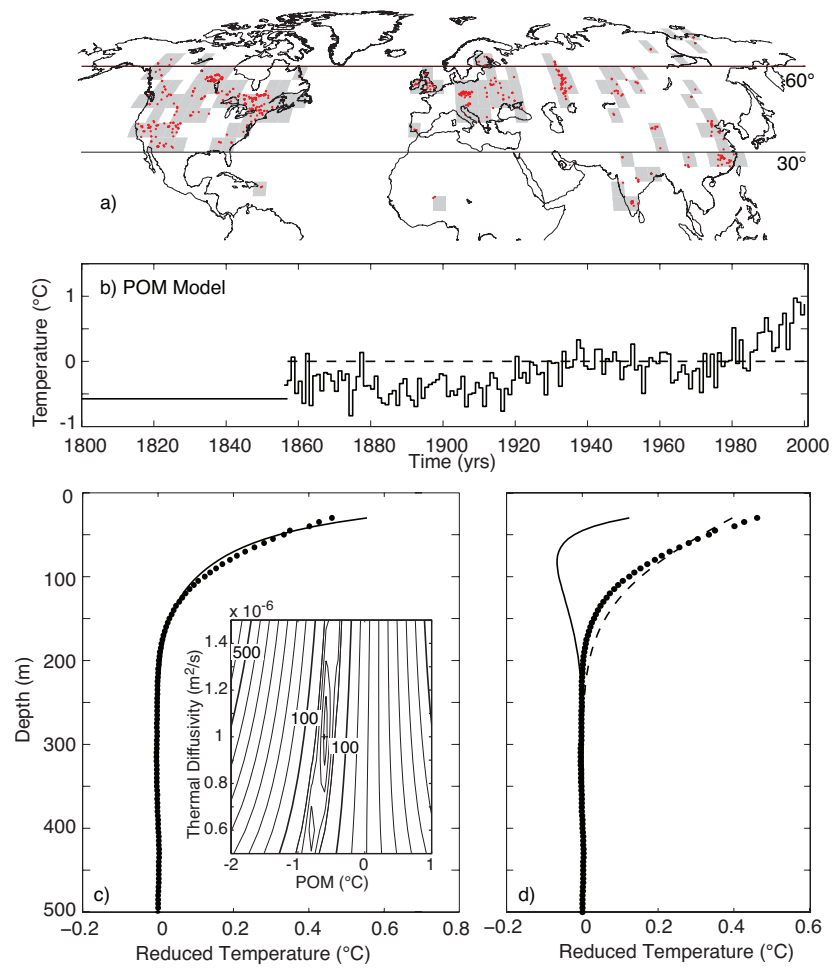

Fig. 6. Extratropical Northern Hemisphere surface air temperature record and average reduced temperature profile. (a) Location map showing boreholes (red circles) and gridded surface air temperature data. (b) Average SAT record relative to 1961-1990 mean temperature. Horizontal line shows $P O M$. (c) Average reduced temperature profile (circles) and best fitting model based on POM and SAT record. Inset shows $R M S$ misfit as a function of $P O M$ and thermal diffusivity. The contour interval is $50 \mathrm{mK}$. (d) Average reduced temperature profile and components of POM model. Solid line shows diffused forcing function and dashed line shows step function.

extrapolates to an amplitude of $0.8^{\circ} \mathrm{C}$ at the surface. The residual misfit of the average reduced temperature profile below $160 \mathrm{~m}$ is $5 \mathrm{mK}$, indicating little if any signal below this depth. This profile represents the diffused GST history at the Earth's surface over the past several centuries.

While linear trends are an oversimplification of both the SAT record and the reduced temperature profile, the trend represents a long period component of each data set, and it is worth noting that both records are optimally fit with a linear increase in temperature of $0.8^{\circ} \mathrm{C}$. In the case of the reduced temperature profile the best fit is over 160 years, but the onset time is poorly resolved because of diffusion. The independent agreement of these trends suggests that the firstorder temperature changes represented by these datasets are consistent. 

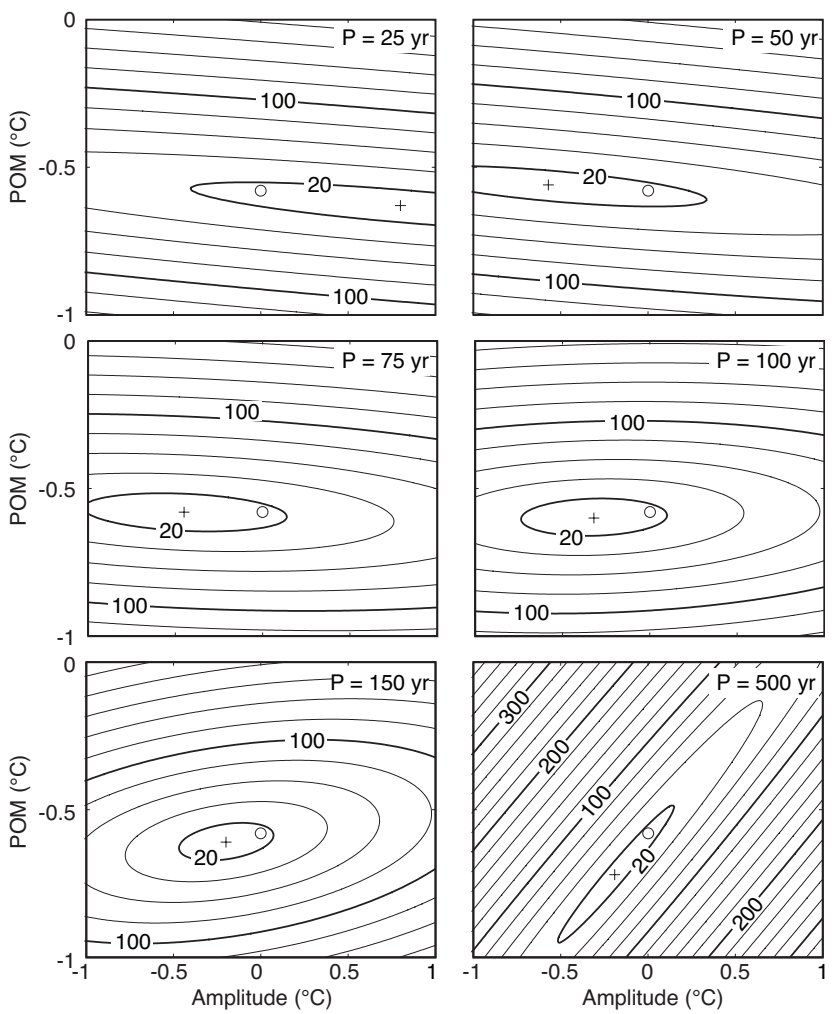

Fig. 7. $R M S$ misfit as a function of forcing amplitude and $P O M$ for different forcing periods. Open circles show observed amplitude and crosses shown optimum amplitude (Table 1). Contour interval is $20 \mathrm{mK}$. Period, $P$, corresponding to each panel is shown in upper right corner.

\section{Northern Hemisphere air and ground temperature tracking}

The Northern Hemisphere SAT record is diffused into the subsurface using Eq. (2) (Fig. 6c). The best fitting model jointly fitting the SAT and reduced temperature profile yields a $P O M$ of $0.58^{\circ} \mathrm{C}$ below the $1961-1990$ mean SAT and a thermal diffusivity of $1 \times 10^{-6} \mathrm{~m}^{2} / \mathrm{s}$ (Fig. 6c, inset). The $R M S$ misfit as a function of the $P O M$ and thermal diffusivity shows that the POM-SAT model is very sensitive to the POM whereas it is relatively insensitive to the choice of thermal diffusivity. Part of the explanation for the lack of sensitivity to the thermal diffusivity is that it enters the equation in the same way time does Eq. (2). The combination of this POM with the last 150 years of SAT data yield a transient profile that is an excellent fit to the observations with a $R M S$ misfit of $18 \mathrm{mK}$.

It is interesting to decompose the POM-SAT model into its two component parts, the forcing function with no degrees of freedom and a step function with the amplitude of the step being the free parameter. The step function represents the null SAT change hypothesis and its subsurface manifestation shows that relative to the average reduced temperature pro-
Table 2. Amplitudes and POMs providing the best fit between the average Northern Hemisphere SAT and reduced temperature profile.

\begin{tabular}{llll}
\hline Period yr & Amplitude ${ }^{\circ} \mathrm{C}$ & $\triangle P O M^{\circ} \mathrm{C}$ & $\triangle R M S \mathrm{mK}$ \\
\hline 25 & -0.80 & -0.03 & 2 \\
50 & 0.64 & 0.02 & 6 \\
75 & 0.32 & -0.02 & 13 \\
100 & 0.32 & -0.02 & 13 \\
150 & 0.20 & -0.03 & 10 \\
500 & 0.20 & -0.14 & 5 \\
\hline
\end{tabular}

Amplitude is the amplitude that optimizes the fit for that period. $\triangle P O M$ is the change in pre-observational mean between the comparison using averaged data and that with the optimum amplitude. $\triangle R M S$ is the change in root mean square misfit using averaged data and that with the optimum amplitude.

file warming starts too early and then does not warm enough in the recent past (Fig. 6d). The diffused version of the SAT record shows the impact of cooler temperatures during the late 1800 's through the early 1900 's and then the rapid warming since the 1980's.

The numerical tests described above suggest a method for investigating relationships between air and ground temperatures at periods longer than are commonly available at borehole climate observatories. Sensitivity of the POM-SAT model for periods corresponding to 25, 50, 75, 100, 150, and 500 years are investigated by adding these signals as a function of amplitude to the SAT record and diffusing it into the ground. Here I ignore potential phase shifts. The fit between the synthetic and reduced temperature profile computed as a function of forcing amplitude and POM (Fig. 7). Consistent with the numerical experiments, there is little sensitivity to errant amplitudes for periods of 50 years and less as indicated by the $R M S$ misfit plots. However, there is good sensitivity at the longer periods investigated as indicated by the well-defined minima in the $R M S$ misfits. For the periods investigated changes in $R M S$ misfit are generally small (Table 2). However, both the 75 and 100-year periods indicates adding heat to the subsurface corresponding to amplitudes of $0.5^{\circ}$ and $0.4^{\circ} \mathrm{C}$, respectively, can decrease the $R M S$ misfit by $13 \mathrm{mK}$. Note however that the change in $P O M$ would be negligible. In contrast adding $0.2^{\circ} \mathrm{C}$ with a period of 500-years would only decrease the $R M S$ misfit by $5 \mathrm{mK}$.

It is interesting that in all cases, except the 25 -year period where sensitivity is minimal, the minimum RMS misfit is obtained by increasing the amplitude of the surface forcing, or conversely decreasing the subsurface heat content. This result suggests that some process may be decoupling air and ground temperatures in such a way as to attenuate heat diffusing into the ground. Further these results suggest that the decoupling process has a dominant period of in the range of 75 to 150 -years. With the exception of the 500-year period, changes to the $P O M$ are negligible. Thus, even though we 
can achieve a smaller $R M S$ misfit by adding heat to the subsurface the overall impact on the POM, and thus estimates of total warming, remains unchanged.

\section{Discussion}

This study addresses two issues arising from the use of the POM-SAT model. The first issue concerns what the model fit says about the consistency between air and ground temperatures. Sensitivity tests (Fig. 5a) indicate that terms like consistency or tracking are most illuminative when discussed in the context of the periods being considered. As implemented in this study the $R M S$ misfit is relatively insensitive to forcing periods shorter than about 50 years. However the POM-SAT model is sensitive to longer periods. Sensitivity to shorter periods may be increased by using data shallower than available in the global database of borehole temperature for climatic reconstruction (Huang et al., 1988), and possibly by truncating the calculation of the $R M S$ at a shallower depth than used in this study. A small RMS misfit as a function of forcing amplitudes at particular periods (e.g. Fig. 7) implies that the reduced temperature profile and forcing function are internally consistent for these periods. In contrast, large $R M S$ misfits may be indicative of 1) significant non-diffusive heat transfer such that a purely diffusive model is inappropriate; 2) a significant thermal event prior to the start of the SAT time series; and 3) a changing relationship between air and ground temperatures. If a significant thermal event prior to the start of the SAT time series is suspected, adding additional parameters prior to the start of the forcing function, which might take the form of step changes in temperature, may be warranted (e.g., Harris and Gosnold, 1999). The significance of additional free parameters can be checked with an F-test. A poor fit between air and ground temperatures offers the exciting possibility of better understanding processes that influence coupling. Land cover change is one such potential process.

The second issue is the robustness of the POM. The POM appears to be a robust parameter as long as errant amplitudes are not occurring at periods much longer than the forcing function. Physically this parameter represents the mean temperature prior to the beginning of the forcing function and is robust because it represents a relatively long time period, i.e., the beginning of the forcing function to approximately $500 \mathrm{ybp}$. This is the standard tradeoff between parameter resolution and variance.

An underlying issue in the use the use of the POM-SAT model is causality between variations in air and ground temperatures. The RMS misfit function is a statistical measure and does not prove air and ground temperatures are tracking other. While it is conceivable that air and ground temperatures show a similar history of variation due to processes independent of each other, it seems more likely that variations in ground and air temperatures are indeed related. This view has support in other comparisons of air and ground temperatures (e.g., Pollack and Smerdon, 2004), at borehole climate observatories (e.g., Putnam and Chapman, 1996; Bartlett et al., 2006) and in comparisons of detailed tracking between air and ground temperatures (e.g., Smerdon et al., 2003, 2004, 2006).

One potential decoupling process of considerable interest is snow cover. It is interesting to note that the results of this study are consistent with the impact of snow cover in midlatitude areas of North America (Bartlett et al., 2005). In this scenario, part of the long-term cold season warming signal is attenuated as it passes through the snow cover and does not reach the ground. As indicated by the sensitivity tests, at these periods and spatial scale the effect of snow cover on the POM is appears to be small. Bartlett et al. (2005) reached a similar conclusion.

Climate analysis based on borehole temperature profiles and proxy records are natural complements of each other. The strength of the temperature profile technique lies in the resolution of temperature at long time-scales. While information concerning the timing of events is contained in temperature profiles, the frequency-depth dependent attenuation of thermal waves leads to temporal resolution that is less than ideal. In contrast annually resolved proxy records such as tree-rings have excellent temporal resolution, but uncertainty stemming from the use of these proxy records at time scales longer than a century is larger than ideal (e.g. Cook et al., 1995; Juckes et al., 2006). In general, early multiproxy records of Northern Hemispheric temperature change showed about $0.5^{\circ} \mathrm{C}$ warming over the past 500 years (e.g., Jones et al., 1988; Mann et al., 1999; Crowley and Lowery, 2002; Briffa et al., 2001). However, recent multiproxy reconstructions which have focused on low frequency variability (e.g., Esper et al., 2002; Moberg et al., 2005; Hegerl et al., 2007) are now in general agreement with temperature profile reconstructions (Huang et al., 2000; Harris and Chapman, 2001; Beltrami, 2002) and indicate about $1{ }^{\circ} \mathrm{C}$ of warming over the past 500 years. The low frequency temperature content of borehole temperature reconstructions can be potentially used as a check against centennial to millennial scale variability now being extracted from proxy records (e.g., Hegerl et al., 2007). However, processes affecting the degree to which air and ground temperatures faithfully track each other are particularly important to understand if information from temperature profiles is going to be incorporated with proxy reconstructions.

Two important extensions to this study include developing an inverse model to simultaneously invert for optimal periods and amplitudes that would bring the results of Table 2 into focus. Secondly, proxy records represent an important extension of surface temperatures into the past, and similar comparisons could be made with proxy records. 


\section{Conclusions}

On the basis of this analysis I conclude the following:

1. Consistent with all diffusive processes, high frequency components of GST variations are attenuated at relatively shallow depths. The POM-SAT model described and implemented here is relatively insensitive to periods shorter than about 50 years.

2. The POM-SAT model has two components, the POM temperature and the forcing function. Both of these components influence the $R M S$ misfit. An additional free parameter is the choice of thermal diffusivity, but the POM-SAT model is relatively insensitive to this parameter.

3. Sensitivity of the RMS misfit and the POM to the forcing function increase as the period of forcing increases up to the 150 year time span of the forcing function investigated in this study.

4. The Northern Hemisphere extratropical average reduced temperature profile compares well with an average SAT record relative to the null SAT hypothesis. The POM is $-0.6^{\circ} \mathrm{C}$ below the $1961-1990$ mean SAT and the $R M S$ misfit is $18 \mathrm{mK}$.

5. Processes decoupling air and ground temperature changes may be present. These changes tend to attenuate observed SAT warming in the ground, and are consistent with the effect of snow muting cold season warming. This effect has a negligible influence on the POM.

Acknowledgements. I thank J. Smerdon for helpful discussions at the seminal stage of this study. Two anonymous reviewers and V. Rath helped to clarify my thinking and improve this paper. This work is supported by NSF-0126029.

Edited by: V. Rath

\section{References}

Baker, J. M. and Baker, D. G.: Long-term heat flux and heat storage at a mid-latitude site, Clim. Change, 54, 295-303, 2002.

Baker, D. G. and Ruschy, D. L.: The recent warming in Eastern Minnesota shown by ground temperatures, Geophys. Res. Lett., 23, 371-374, 1993.

Bartlett M. G., Chapman, D. S., and Harris, R. N.: Snow effect on North American ground temperatures, 1950-2002, J. Geophys. Res. 110, doi:10.1029/2005JF000293, 2005.

Bartlett, M. G., Chapman, D. S., and Harris, R. N.: A decade of ground-air temperature tracking at Emigrant Pass Observatory, Utah, J. Climate, 19, 3722-3731, 2006.

Beltrami, H: Climate from borehole data: Energy fluxes and temperatures since 1500, Geophys. Res. Lett., 29, 2111, doi:10.1029/2002GL015702, 2002.

Beltrami, H. and Mareschal, J. C.: Resolution of Ground Temperature Histories Inverted from Borehole Temperature Data, Global and Planet. Change, 11, 57-70, 1995.

Beltrami, H., Smerdon, J., Pollack, H. N., and Huang, S.: Continental heat gain in the global climate system, Geophys. Res. Lett., 29, 1167, doi:10.1029/2001GL014310, 2002.
Briffa, K. R., Osborn, T. J., Schweingruber, F. H., Harris, I. C., Jones, P. D., Shiyatov, S. G., and Vaganov, E. A.: Low frequency temperature variations from a northern tree-ring density network, J. Geophys. Res., 106, 2929-2941, 2001.

Carslaw H. S. and Jaeger J. C.: Conduction of Heat in Solids. Oxford, UK: Oxford Univ. Press, 510 pp., 2nd ed., 1959.

Chisholm, T. J. and Chapman, D. S.: Climate change inferred from analysis of borehole temperatures: an example from western Utah, J. Geophys. Res., 97, 14 155-14 175, 1992

Chudninova, S. M., Frauenfeld, O. W., Barry, R. G., Zhang, T., and Sorokovikov, V. A.: Relationship between air and soil temperature trends and periodicities in the permafrost regions of Russia, J. Geophys. Res., 111, F02008, doi:10.1029/2005JF000342, 2006.

Clauser, C. and Huenges, E. L.: Thermal conductivity of rock and minerals, in: Rock Physics and Phase Relations: A Handbook of Physical Constants, Ref. Shelf Ser., vol 3, edited by: Ahrens, T. J., pp. 105-126, Washington D. C., 1995.

Clow, G.: Temporal resolution of surface-temperature histories inferred from borehole temperature measurements, Global Planet. Change, 98, 81-86, 1992.

Cook, E. R., Briffa, K. R., Meko, D. M., Graybill, D. S., and Funkhouser, G.: The "segment length curse" in long treering chronology development for paleoclimatic studies, The Holocene, 5, 229-237, 1995.

Crowley T. J. and Lowery, T. S.: How warm was the Medieval warm period?, Ambio, 29, 51-54, 2002.

Esper, J., Cook, E. R., and Schweingruber, F. H.: Low-frequency signals in long tree-ring chronologies for reconstructing past temperature variability, Science, 295, 2250-2253, 2002.

García-Suárez, A. M. and Butler, C. J.: Soil temperatures at Armagh Observatory, Northern Ireland, from 1904 to 2002, Int. J. Clim., 26, 1075-1089, 2006.

González-Rouco, J. F., Beltrami, H., Zorita, E., and von Storch, H.: Simulation and inversion of borehole temperature profiles in surrogate climates: Spatial distribution and surface coupling, Geophys. Res. Lett., 33, L01703, doi:10.1029/2005GL024693, 2006.

Gonzalez-Rouco, J. F., von Storch, H., and Zorita E.: Deep soil temperature as proxy for surface air-temperature in a coupled model simulation of the last thousand years, Geophys. Res. Lett., 30, 2116-2119, 2003.

Harris, R. N. and Chapman, D. S.: Geothermics and climate change: Part 1, Analysis of borehole temperatures with emphasis on resolving power, J. Geophys. Res, 103, 7363-7370, 1998a.

Harris, R. N. and Chapman, D. S.: Geothermics and climate change: Part 2, Joint analysis of borehole temperatures and meteorological data, J. Geophys. Res., 7371-7383, 1998b.

Harris, R. N. and Chapman, D. S.: Mid-Latitude $\left(30^{\circ}-60^{\circ} \mathrm{N}\right)$ climatic warming inferred by combining borehole temperatures with surface air temperatures, Geophys. Res. Lett., 28, 747-750, 2001.

Harris, R. N. and Chapman, D. S.: Borehole temperatures and treerings: Seasonality and estimates of extratropical Northern Hemispheric warming, J. Geophys. Res., doi:10.1029/2005JF000303, 2005.

Harris, R. N. and Gosnold, W. D.: Comparisons of borehole temperature-depth profiles and surface air temperatures in the northern plains of the U.S., Geophys. J. Int., 138, 541-548, 1999. 
Hegerl, G. C., Crowley, T. J., Allen, M., Hyde, W. T., Pollack, H. N., Smerdon, J. E., and Zorita, E.: Detection of human influence on a new, validated 1500-year temperature reconstruction, J. Climate, 20, 650-666. 2007.

Huang, S. and Pollack, H. N.: Global Borehole Temperature Database for Climate Reconstruction (IGBP PAGES/Word Data Center-A for Paleoclimatology Data Contribution Series No. 1998-044, NOAA/NGDC Paleoclimatology Program Boulder, Colorado, 1998).

Huang, S. Pollack, H. N., and Shen, P. Y.: Temperature trends over the past five centuries reconstructed from borehole temperatures, Nature, 403, 756-758, 2000.

Jones, P. D., Briffa, K. R., Barnett, T. P., and Tett, S. F. B.: Highresolution palaeoclimatic records for the last millennium: interpretation, integration and comparison with General Circulation Model control-run temperatures, The Holocene, 8, 455-471, 1998.

Jones, P. D. and Moberg, A.: Hemispheric and large-scale surface air temperature variations: an extensive revision and an update to 2001, J. Climate, 16, 206-223, 2003.

Juckes, M. N., Allen, M. R., Briffa, K. R., Esper, J., Hegerl, G. C., Moberg, A., Osborn, T. J., Weber, S. L., and Zorita, E., Millennial temperature reconstruction intercomparison and evaluation, Clim. Past, 2, 1001-Clim. Past Discuss., 2, 1001-1049, 2006, www.clim-past-discuss.net/2/1001/2006/.

Lachenbruch, A. H. and Marshall, B. V.: Changing climate; geothermal evidence from permafrost in the Alaskan Arctic. Science 23, 689-696, 1986.

Lewis T. J. and Wang, K.: Geothermal evidence for deforestation induced warming: implications for the climatic impact of land development, Geophys. Res. Lett., 25, 535- 538, 1998.

Mann, M. E., Bradley, R. S., and Hughes, R. S.: Northern hemisphere temperatures during the past millennium: inferences, uncertainties and limitations, Geophys. Res. Lett., 26, 759-762, 1999.

Mitchell, T. D. and Jones, P. D. : An improved method of constructing a database of monthly climate observations and associated high-resolution grids, Inter. J. Clim., 25, doi:10.1002/joc.1181 693-712, 2005.

Moberg, A., Sonechkin, D. M., Holmgren, K., Datsenko N. M., and Karlen, W.: Highly variable Northern Hemisphere temperatures reconstructed from low- and high-resolution proxy data, Nature, 433, doi:10.1038/nature03265, 2005.

Mottaghy, D. and Rath, V.: Latent heat effects in subsurface heat transport modeling and their impact on palaeotemperature reconstructions, Geophys. J. Int., doi:10.1111/j.1365246X.2005.02843.x, 2005.

Nitoiu, D. and Beltrami, H.: Subsurface thermal effects of land use changes, J. Geophys. Res., 110, doi:10.1029/2004JF000151, 2005.

Pollack, H. N. and Huang, S.: Climate reconstruction from subsurface temperatures, Ann. Rev. Earth Planetary Sci., 28, 339-365, 2000.

Pollack, H. N., Smerdon, J. E., and van Keken, P. E.: Variable seasonal coupling between air and ground temperatures: A simple representation in terms of subsurface thermal diffusivity, Geophys. Res. Lett., 32, L15405, doi:10.1029/2005GL023869, 2005.

Pollack, H. N. and Smerdon, J. E.: Borehole climate reconstructions: Spatial structure and hemispheric averages, J. Geophys.
Res., 109, D11106, doi:10.1029/2003JD004163, 2004.

Putnam S. N. and Chapman, D. S.: A geothermal climate-change observatory: first year results from Emigrant Pass Observatory in northwest Utah, J. Geophys. Res., 101, 21 877-21 890, 1996.

Smerdon, J. E., Pollack, H. N., Cermak, V., Enz, J. W., Kresl, M., Safanda, J., and Wehmiller, J. F.: Air-ground temperature coupling and subsurface propagation of annual temperature signals, J. Geophys. Res., 109, D21107, doi:10.1029/2004JD005056, 2004.

Smerdon, J. E., Pollack, H. N., Enz, J. W., and Lewis, M. J.: Conduction-dominated heat transport of the annual temperature signal in soil, J. Geophys. Res., 108(B9), 2431, doi:10.1029/2002JB002351, 2003.

Smerdon, J. E., Pollack, H. N., Cermak, V., Enz, J. W., Kresl, M., Safanda, J., and Wehmiller, J. F.: Daily, seasonal and annual relationships between air and subsurface temperatures, J. Geophys. Res., 111, D07101, doi:10.1029/2004JD005578, 2006.

Stieglitz, M. and Smerdon, J. E.: Characterizing land-atmosphere coupling and the implications for subsurface thermodynamics, J. Clim., doi:10.1175/JCLI3982.1, 21-37, 2007.

Taylor, A. E., Wang, K., Smith, S. L., Burgess, M. M., and Judge, A. S.: Canadian Arctic Permafrost Observatories: Detecting contemporary climate change through inversion of subsurface temperature time series, J. Geophys. Res., 111, B02411, doi:10.1029/2004JB003208, 2006. 